\title{
Phase-Shifting Control of Double Pulse in Harmonic Elimination Wei Peng ${ }^{1, a^{*}}$, Junhong Zhang ${ }^{1}$, Jianxin gao ${ }^{1, b}$, Guangyi $\mathrm{Li}^{1, \mathrm{c}}$ \\ ${ }^{1}$ College of Electrical Engineering, Naval Univ. of Engineering, Wuhan 430033, China \\ a1530911613@qq.com, bgaojianxin_cn@163.com, ${ }^{d} 530785601 @ q q . c o m$
}

\section{Keywords: Double pulse, Phase-shifting control, FFT analysis}

\begin{abstract}
With the wide usage of electronic devices, harmonic wave is becoming worse. A method of Phase-Shifting Control of Double Pulse (PSCDP) is introduced in this paper. In continuous conduction mode, mathematical model between harmonic and phase-shifting is built, FFT is used to evaluate the harmonic content under different phase-shifting. The result indicates that the method of PSCDP can reduce output harmonic and make the DC-DC converter less dependent on filter.
\end{abstract}

\section{Introduction}

DC-DC converter, with many excellent properties like high power convert efficiency, high power density, is widely used in the motor speed. However, power electronic devices' switching operations will generate harmonics and influence the running of the motor. Although active power filter has outstanding effect on harmonic elimination, the design is complex and the cost is high. Passive filter is widely used in practical engineering, for its easy design and low cost. But the passive filter is not flexible, a set of LC filter can only eliminate a certain order harmonic. To reach a great effect on suppressing the converter's output harmonics, many groups of LC filter is needed.

If the harmonic waves generated in power electronic devices' switching operations is reduce, the output is less dependent on filters.

Phase-Shifting Control of Double Pulse (PSCDP) is the improvement of PWM (Pulse Width Modulation). The paper shows how Phase-Shifting Control of Double Pulse can reduce harmonic waves when used in Boost circuit. In the PSCDP method, two pulses of different duty cycle but the same frequency are used to control the switching operations of power electronic devices. The output wave is dependent on the duty cycle of each pulse and phase difference between them. When the pulses are at appropriate duty cycle and phase, the harmonic wave is lower.

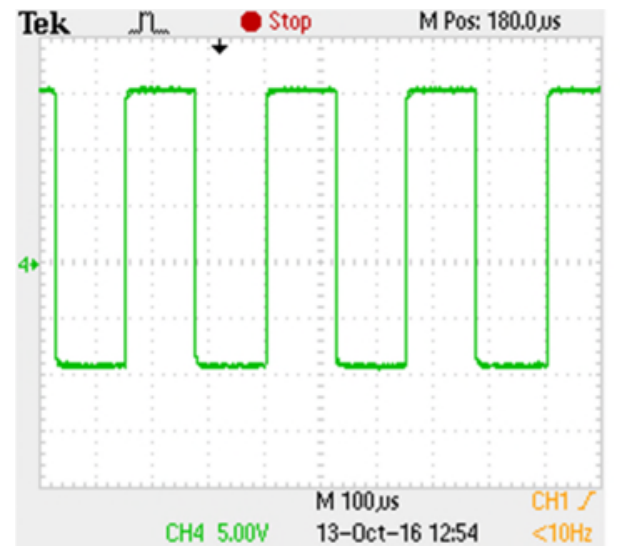

a. Ordinary PWM signal

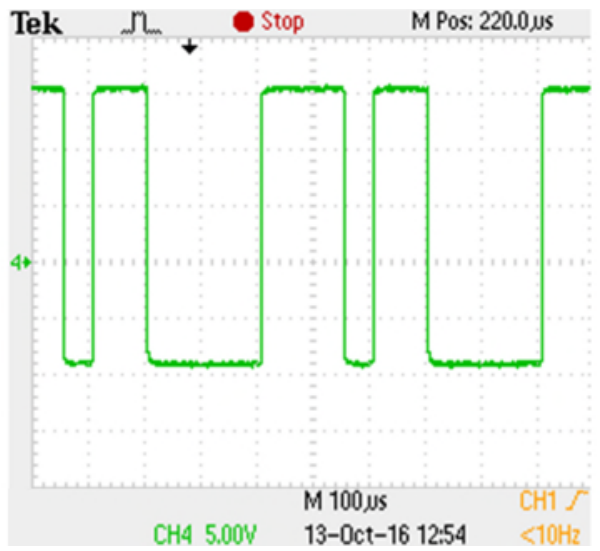

b. Double PWM signal

Fig. 1 The compacyclen graph of ordinary PWM and double PWM signal.

Fig. 1 shows the difference between the single pulse-width signal (on left part) and double pulse-width signal (on right part). When the total duty cycle of the two pulses are the same with single pulse, the average output voltage will be the same. 


\section{The Working Principle of Boost Circuit Controlled by Single Pulse}

On Current Continuous Mode, the work period of Boost circuit can be divided into two steps. Fig. 2 shows the working mechanism of Boost circuit controlled by single and double pulse.

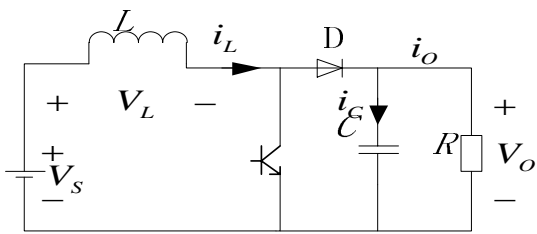

a. Boost circuit

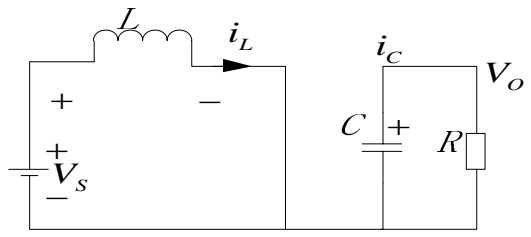

b. $T$ is on, $D$ is off

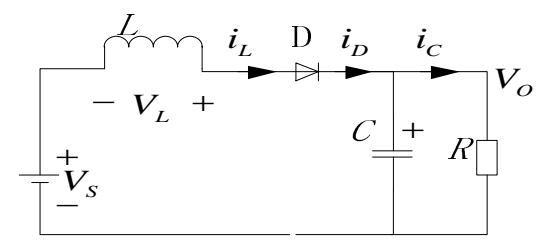

c. $\mathrm{T}$ is off, $\mathrm{D}$ is on

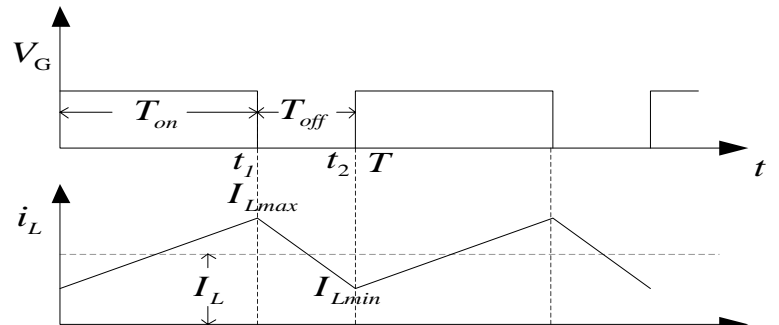

d. Waveform controlled by single pulse

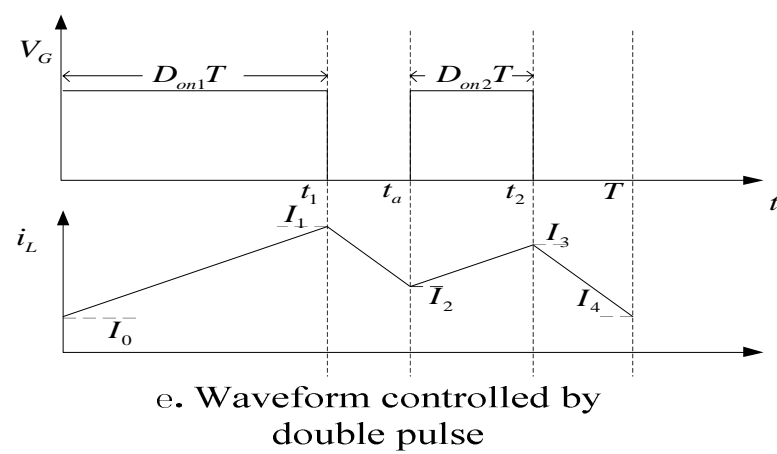

Fig. 2 The working mechanism of Boost circuit.

1)Just as Fig. 2.a shows, when the switching tube is on, the voltage change of capacitor is not great, and voltage on inductor is $V_{S}$, so the inductor current grows linearly. When $t=T_{o n}=D T_{S}, i_{L}$ comes to the largest $I_{\max }$. During time $T_{o n},{ }_{L}$ has increased $i_{L+}$ :

$$
i_{L+}=\frac{V_{S}}{L} T_{o n}=\frac{V_{S}}{L} D T_{S}
$$

2) When the switching tube is off, the voltage of capacitor is not great, the voltage on inductor is stable at $V_{S}-V_{O}$, for $V_{S}<V_{O}, i_{L}$ decreases linearly, $L \bullet d i_{L} / d t=V_{S}-V_{O}$. During time $T_{\text {off }}$, $i_{L}$ has decreased $i_{L_{-}}$:

$$
i_{L-}=\frac{V_{O}-V_{S}}{L} T_{o f f}=\frac{V_{O}-V_{S}}{L}(1-D) T_{S}
$$

When the circuit is stable, the inductor increase and decrease should be the same:

$$
\begin{aligned}
& i_{L+}=\frac{V_{S}}{L} D T_{S}=i_{L-}=\frac{V_{O}-V_{S}}{L}(1-D) T_{S} \\
& V_{O}=\frac{V_{S}}{1-D}
\end{aligned}
$$

The average output voltage is $V_{O}, V_{O}$ ranges between $V_{\text {Omin }}$ and $V_{O \max }$, the output voltage is: 


$$
v_{O}(t)=\left\{\begin{array}{l}
V_{\text {Omax }}\left(1-\frac{t}{R C}\right) ; \quad 0 \leq t \leq D T_{S} \\
V_{\text {Omin }}+\frac{D V_{O}}{R C(1-D)}\left(t-D T_{S}\right) ; \quad D T_{S} \leq t \leq T_{S}
\end{array}\right.
$$

Switch to the frequency domain and get fourier transformed:

$$
\begin{aligned}
& v_{O}(\omega t)=\left\{\begin{array}{l}
V_{\text {Omax }} \bullet\left(1-\frac{\omega t}{R C}\right) ; 0 \leq \omega t \leq 2 \pi D \\
V_{\text {Omin }}+\frac{D V_{O}}{(1-D)} \frac{(\omega t-2 \pi D)}{R C} ; 2 \pi D \leq \omega t \leq 2 \pi
\end{array}\right. \\
& v_{O}(\omega t)=V_{O}+\sum_{n=1}^{\infty}\left[a_{n} \cos (n \omega t)+b_{n} \sin (n \omega t)\right]
\end{aligned}
$$

(6)

$$
a_{n}=\frac{V_{O}}{n^{2} \pi \omega R C(1-D)}[1-\cos (2 n \pi D)] \quad b_{n}=-\frac{V_{O}}{n^{2} \pi \omega R C(1-D)} \sin (2 n \pi D)
$$

It can be concluded from Eq. 6 that the output harmonic is related to the frequency and duty cycle under certain circuit parameter. Increase the working frequency can reduce output harmonic wave, but may lead to great switching loss.

\section{The Working Principle of Boost Circuit Controlled by Double Pulse}

The first pulse duty cycle is $D_{o n 1}$, the second is $D_{o n 2}$, switching period is $T$, total duty cycle is $D$, just as Fig. 2.e shows, their relationships are listed in equation 2-0 1, when $M, N$ and total duty cycle is set,each pulse duty cycle and phase difference are set:

$$
\left\{\begin{array}{l}
T_{o n 1}=D_{o n 1} \times T \\
T_{o n 2}=D_{o n 2} \times T=N D_{o n 1} \times T \\
D=D_{o n 1}+D_{o n 2} \\
D_{o n 2} T+t_{a} \leq T \\
t_{a}=M D_{o n 1} T \geq D_{o n 1} T
\end{array}\right.
$$

1) When the switching tube is on under the control of the first pulse, the capacitor powers the load $R$, the capacity voltage $\left(v_{O}(t)\right)$ decreases linearly:

$$
v_{O}(t)=V_{0} \bullet\left(1-\frac{t}{R C}\right)
$$

When $t=T_{o n 1}=D_{o n 1} T, v_{O}(t)$ comes to $V_{1}, v_{O}(t)$ has totally decreased $\Delta V_{-1}$.

$$
\begin{aligned}
& \Delta V_{-1}=V_{O} \frac{D_{o n 1} T_{S}}{R C} \\
& V_{1}=V_{0} \bullet\left(1-\frac{D_{o n 1} T_{S}}{R C}\right)
\end{aligned}
$$

2) When the switching tube is off under the control of the first pulse, the capacitor is charged, the capacity voltage $\left(v_{O}(t)\right)$ increases linearly: 
$v_{O}(t)=V_{1}+\frac{D V_{O}}{R C(1-D)} t$

When $t=M D_{o n 1} T_{S}, v_{O}(t)$ increases to $V_{2}, v_{O}(t)$ has totally increased $\Delta V_{+1}$,

$$
\begin{gathered}
\Delta V_{+1}=\frac{D V_{O}}{R C(1-D)}(M-1) D_{o n 1} T_{S} \\
V_{2}=V_{1}+\frac{D V_{O}}{R C(1-D)}(M-1) D_{o n 1} T_{S}
\end{gathered}
$$

When the switching tube is on under the control of the second pulse, the capacitor powers the load $R$, the capacity voltage $\left(v_{O}(t)\right)$ decreases linearly:

$$
v_{O}(t)=V_{2} \bullet\left(1-\frac{t}{R C}\right)
$$

When $t=D_{o n 2} T+M D_{o n 1} T, v_{O}(t)$ comes to $V_{3}, v_{O}(t)$ has totally decreased $\Delta V_{-2}$ :

$$
\begin{gathered}
\Delta V_{-2}=V_{O} \frac{D_{o n 2} T_{S}}{R C} \\
V_{3}=V_{2} \bullet\left(1-\frac{D_{o n 2} T_{S}}{R C}\right)
\end{gathered}
$$

3) When the switching tube is off under the control of the first pulse, the capacitor is charged, the capacity voltage $\left(v_{O}(t)\right)$ increases linearly:

$$
v_{O}(t)=V_{3}+\frac{D V_{O}}{R C(1-D)} t
$$

When $t=T_{S}, v_{O}(t)$ comes to $V_{4}=V_{0}, v_{O}(t)$ has totally decreased $\Delta V_{+2}$ :

$$
\begin{aligned}
& \Delta V_{+2}=\frac{D V_{O}}{R C(1-D)}(M-1) D_{o n 1} T_{S} \\
& V_{4}=V_{3}+\frac{D V_{O}}{R C(1-D)}\left(1-M D_{o n 1}-D_{o n 2}\right) T_{S}
\end{aligned}
$$

The capacity voltage in a period is:

$$
v_{O}(t)=\left\{\begin{array}{l}
V_{0} \bullet\left(1-\frac{t}{R C}\right) ; \quad 0 \leq t \leq D_{o n 1} T_{S} \\
V_{1}+\frac{D V_{O}}{R C(1-D)}\left(t-D_{o n 1} T_{S}\right) ; \quad D_{o n 1} T_{S} \leq t \leq M D_{o n 1} T_{S} \\
V_{2} \bullet\left(1-\frac{t-M D_{o n 1} T_{S}}{R C}\right) ; M D_{o n 1} T_{S} \leq t \leq t_{2} \\
V_{3}+\frac{D V_{O}}{R C(1-D)}\left(t-t_{2}\right) ; \quad t_{2} \leq t \leq T
\end{array}\right.
$$

Switch to the frequency domain and fourier transformed: 


$$
\begin{aligned}
& v_{O}(\omega t)=V_{O}+\sum_{n=1}^{\infty}\left[a_{n} \cos (n \omega t)+b_{n} \sin (n \omega t)\right] \\
& a_{n}=\frac{V_{O}}{n^{2} \pi R C \omega(1-D)}\left[1-\cos \left(2 n \pi \frac{1}{1+N} D\right)+\cos \left(2 n \pi \frac{M}{1+N} D\right)-\cos \left(2 n \pi \frac{M+N}{1+N} D\right)\right] \\
& b_{n}=\frac{V_{O}}{n^{2} \pi R C \omega(1-D)}\left[-\sin \left(2 n \pi \frac{1}{1+N} D\right)+\sin \left(2 n \pi \frac{M}{1+N} D\right)-\sin \left(2 n \pi \frac{M+N}{1+N} D\right)\right]
\end{aligned}
$$

It can be concluded from Eq. 21 that the output harmonic is related to the frequency, $M, N$ under certain circuit parameter. Appropriate $M, N$ can be work out to decrease harmonic wave.

\section{Simulation Analysis and Conclusion}

Models are built in Simulink to study the relationship between output and $M, N$. The working frequency is set at $10000 \mathrm{~Hz}$, the inductor $L$ is $600 \mu \mathrm{H}$, the capacitor $C$ is $330 \mu \mathrm{F}$, the DC voltage source is $100 \mathrm{~V}$, the load $R$ is $5 \Omega$.

Fig. 3 shows the output voltage when the switching tube is controlled by single pulse, and double pulse when $M=5 / 3$ with $N=2 / 3, M=3$ with $N=3 / 2, M=8 / 3$ with $N=7 / 3$. The total duty cycle is 0.5 .

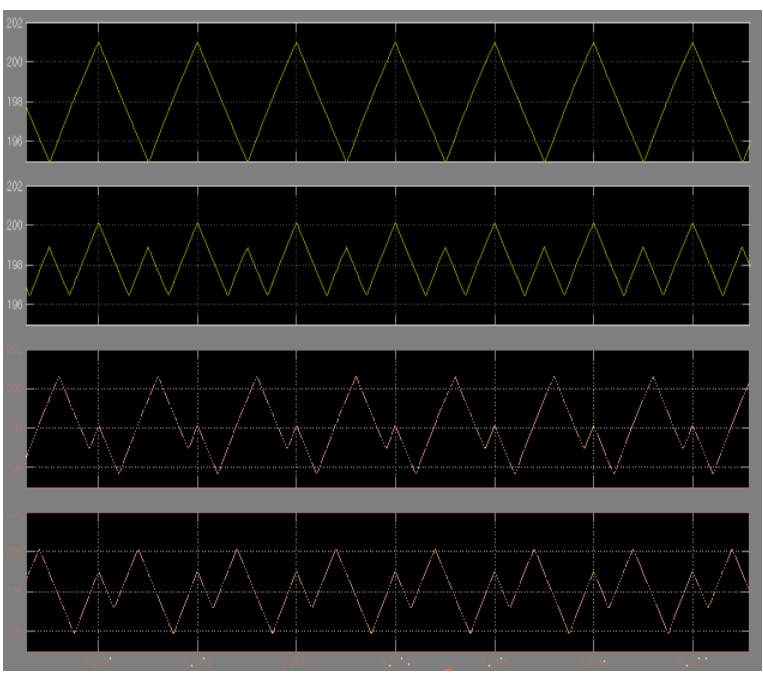

Fig. 3 Output voltage controlled by single and double pulse.

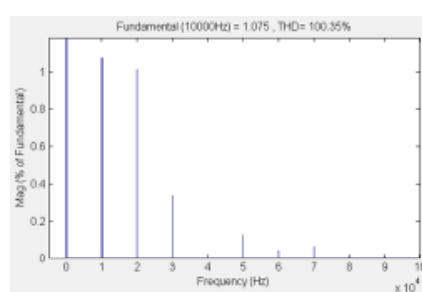

a. $D=0.5$

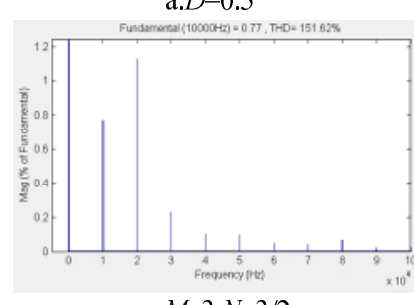

c. $M=3, N=3 / 2$

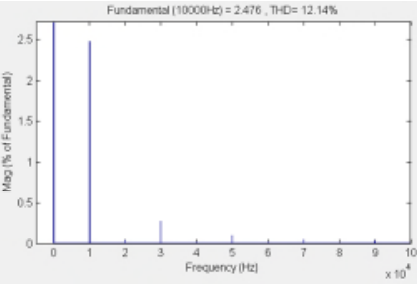

b. $M=5 / 3, N=2 / 3$

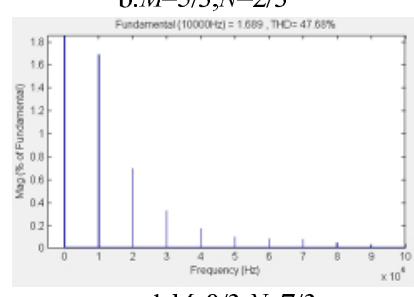

d. $M=8 / 3, N=7 / 3$

Fig. 4 FFT analysis of output harmonic.

It can be concluded from Fig. 3 that PSCDP method can reduce the output voltage ripple.The output changes with the duty cycle of each pulse and the phase difference varying. When $M=5 / 3$ and $N=2 / 3$, the output voltage ripple is smaller than that on the other two conditions.

Fig. 4.b indicates that when $M=5 / 3$ and $N=2 / 3$, the second harmonic is almost reduced to zero. While in Fig. 4.c, the fundamental harmonic is greatly reduced. Combined with Eq. 21, it can be concluded that appropriate $M$ and $N$ can be work out to reduce output harmonic to the greatest degree.

Experiment was conducted to verify the superiority of optimized PSCDP when compared with ordinary PWM, Fig. 5 compares the output voltage waveform and output current waveform, than FFT analysis was made, Fig. 6 shows the amplitude of harmonic under ordinary PWM and PSCDP.

It can be concluded from Fig. 6 that harmonic suppression results under PSCDP was better than ordinary PWM. Fig. 6 the frequency spectrogram of output voltage under ordinary PWM and PSCDP. 


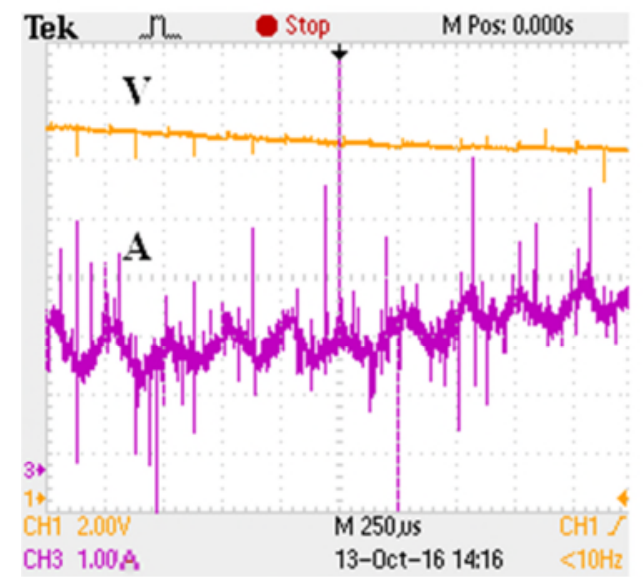

(a) ordinary PWM

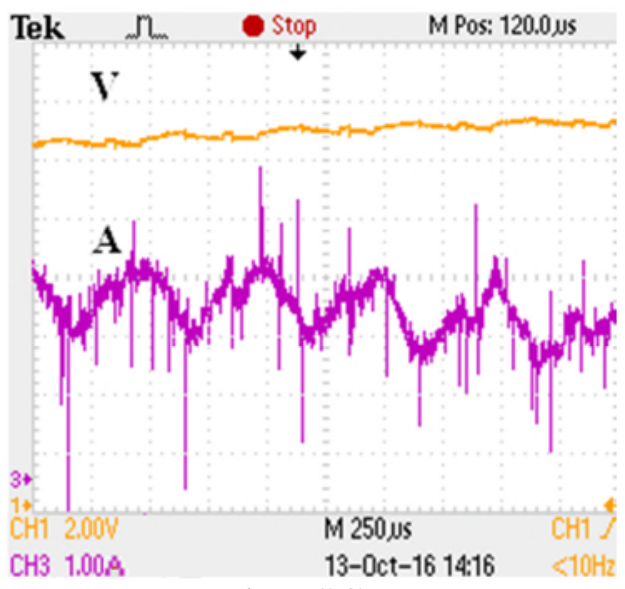

(b) PSCDP

Fig. 5 The comparation of output under PWM and PSDPWM.

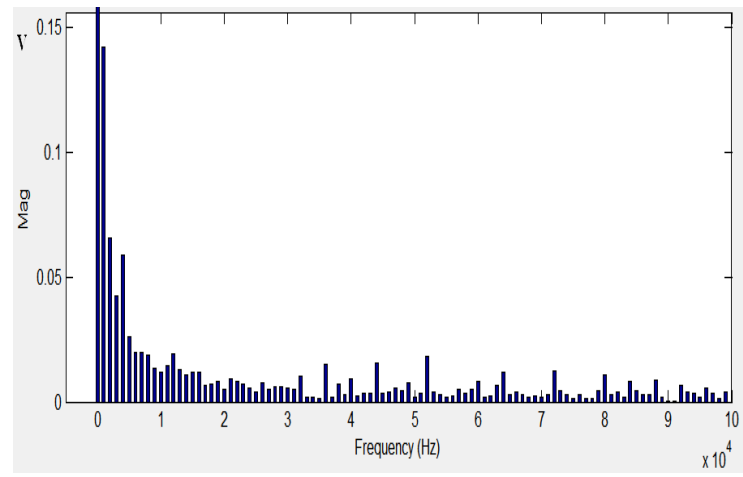

(a) ordinary PWM

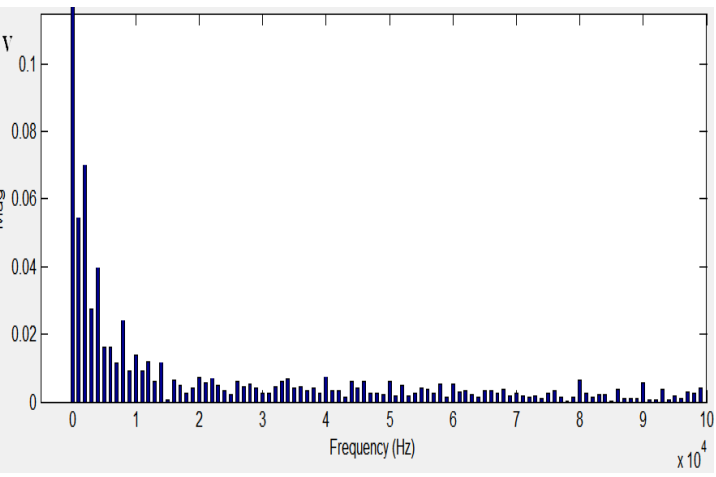

(b) PSCDP

Fig. 6 The frequency spectrogram of output voltage under ordinary PWM and PSCDP.

\section{References}

[1] S. P. Hasmukh, G. H. Richard. Generalized techniques of harmonic elimination and voltage control in thyristor inverters: Part I-Harmonic Elimination. IEEE Trans. Ind. Appl. IA-9(3) (1973).

[2] J. W. Dixon, B. T. Ooi. Indirect current control of a unity power factor sinusoidal current boost type three-phase rectifier. IEEE Trans. Ind. Electron. 1988.

[3] O. Tokun. Three Phase PWM Converter/Inverter by means of Instantaneous Active and Reactive Power Control. Proc. IEEE, IECON, 1991.

[4] M. Tomokazu, N. Mutsuo. Practical Evaluations of a ZVS-PWM DC-DC Converter With Secondary-Side Phase-Shifting Active Rectifier. IEEE Trans. Power Electron. 2011.

[5] Y. M. Jiang, et al. A Novel Single-phase Power Factor Correction Scheme. IEEE. 1993. 